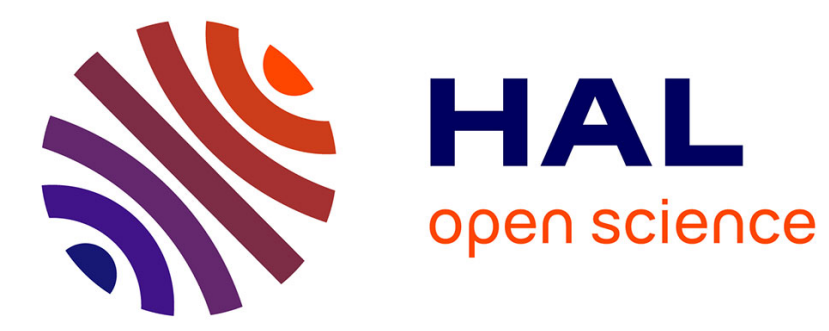

\title{
Handling mixed-state magnetization data for magnetocaloric studiesa solution to achieve realistic entropy behaviour
}

S Das, J S Amaral, V S Amaral

\section{- To cite this version:}

S Das, J S Amaral, V S Amaral. Handling mixed-state magnetization data for magnetocaloric studiesa solution to achieve realistic entropy behaviour. Journal of Physics D: Applied Physics, 2010, 43 (15), pp.152002. 10.1088/0022-3727/43/15/152002 . hal-00569573

\section{HAL Id: hal-00569573 \\ https://hal.science/hal-00569573}

Submitted on 25 Feb 2011

HAL is a multi-disciplinary open access archive for the deposit and dissemination of scientific research documents, whether they are published or not. The documents may come from teaching and research institutions in France or abroad, or from public or private research centers.
L'archive ouverte pluridisciplinaire HAL, est destinée au dépôt et à la diffusion de documents scientifiques de niveau recherche, publiés ou non, émanant des établissements d'enseignement et de recherche français ou étrangers, des laboratoires publics ou privés. 


\title{
Handling mixed state magnetization data for magnetocaloric studies - a solution to achieve realistic entropy behaviour
}

\author{
S. Das ${ }^{1}$, J. S. Amaral ${ }^{1,2}$, V. S. Amaral ${ }^{1}$ \\ ${ }^{1}$ Departamento de Física and CICECO, Universidade de Aveiro, 3810-193 Aveiro, Portugal \\ ${ }^{2}$ IFIMUP-IN and Departamento de Física e Astronomia da Faculdade de Ciências da Universidade do Porto, Rua \\ do Campo Alegre 687, 4169-007 Porto, Portugal \\ Email: soma@ua.pt, jamaral@ua.pt, vamaral@ua.pt
}

\begin{abstract}
We present how to extract a realistic magnetic entropy value from nonequilibrium magnetization data near the transition temperature of a typical first-order system with mixed phase state, influenced by the phase transformation, which is responsible for large values reported, even higher than the theoretical limit. The effect of the mixed phase state is modelled in the magnetization and its nonphysical contribution is removed to obtain the magnetic entropy in accordance with calorimetric experiment and theoretical simulation. This approach gives a reliable estimation of the magnetic entropy value incorporating experimental nonequilibrium magnetization data and correcting the use of Maxwell's relation.
\end{abstract}


Materials with first-order magnetic transition draw attraction because of their reported giant magnetocaloric effect (GMCE) with large peak values of magnetic entropy change $(\Delta S)$ [1-4]. Large entropy values are reported, which are obtained using Maxwell's relation from magnetization measurements. However, these values are often higher than the theoretical limit of pure magnetic contribution [5-12] and are in disagreement with calorimetric measurements, indicating the probable overestimation of entropy [13-16]. The use of Maxwell's relation for a first-order system is questionable for nonequilibrium experimental conditions (usually due to metamagnetic transition) [1317]. A realistic evaluation of entropy was proposed by Amaral et al [17] estimating equilibrium magnetization from experimental nonequilibrium data and using Landau theory/mean field model. Another solution to this problem was presented by Tocado et al [14], who suggested the use of calorimetric measurements and taking temperature dependent magnetization data at constant field to avoid the metamagnetic transition. However, such a procedure is lengthy as temperature stabilization is very time-consuming. An approach to estimate a correct magnetic entropy change using data obtained in common isothermal magnetization measurements is timely required.

Here, we have correlated the effect of the mixed state and phase transformation in a first-order transition system on the magnetic entropy. Such contribution is identified as the cause for the reported discrepancies and can be separated by carefully handling and appropriately modeling the experimental data enabling a close estimation of realistic entropy value.

To justify and exemplify the procedure, we used doped MnAs samples with different mixedphase state characteristics. The compositions, $\mathrm{Mn}_{0.99} \mathrm{Cu}_{0.005} \mathrm{Cr}_{0.005} \mathrm{As}$ (S1), $\mathrm{Mn}_{0.98} \mathrm{Cu}_{0.01} \mathrm{Cr}_{0.01} \mathrm{As}$ (S2) and $\mathrm{Mn}_{0.98} \mathrm{Cu}_{0.015} \mathrm{Cr}_{0.005} \mathrm{As}$ (S3) were prepared through the route used by Rocco et al [6]. The introduction of $\mathrm{Cu} / \mathrm{Cr}$ leads to some changes in $\mathrm{T}_{\mathrm{C}}$, and in a higher extent, to a broadening of the phase transition, which we use to illustrate the physical grounds and reliability of the calculation method.

Phase purity and crystal structure of the samples were confirmed by XRD. Magnetization studies were carried out in a vibrating sample magnetometer (VSM) up to $320 \mathrm{~K}$ and the magnetic field cycling up to 10 Tesla. Magnetic transition temperatures $\left(\mathrm{T}_{\mathrm{C}}\right)$ for these samples are $290-296 \mathrm{~K}$ for cooling and 312-316 K for heating with thermal hysteresis $\sim 18-22 \mathrm{~K}$. The saturation magnetization $\left(\mathrm{M}_{\mathrm{S}}\right)$ at $5 \mathrm{~K}$ is $\sim 3.4 \mu_{\mathrm{B}} / \mathrm{f}$.u. for these samples. Each magnetic isotherm is measured for both increasing 
and decreasing field starting with a pre-history of warming up to $320 \mathrm{~K}$. The typical behaviour obtained is shown in figure 1(a)-(b) up to 5 Tesla with a field cycling up to 10 Tesla. For the increasing field below $\mathrm{T}_{\mathrm{C}}$, the behaviour is ferromagnetic. Above it, in the mixed state, we find the characteristic plateau followed by the metamagnetic transition to the ferromagnetic state at the critical field, as typical for MnAs system [7]. For the decreasing field, no plateau is observed as we drive the experiment above the critical magnetic field for each isotherm. An estimation of the pre-saturation value of the ferromagnetic (FM) phase in the mixed state is obtained from each isothermal magnetization curve for increasing field in the plateau. This gives a direct measure of the FM fraction (x) in the system and is shown in the inset of figure 1(c) as a function of temperature. The derivative of this FM fraction (figure 1(c)) reveals that the chemical doping by Copper and Chromium has introduced different mixed phase characteristics into the system. The higher Chromium content (S2) lowers the transition temperature and broadens the transition zone. However, Copper only influences the mixed phase dynamics. The full widths at half maxima (FWHM) of this phase transition for the samples $\mathrm{S} 1$ and $\mathrm{S} 3$ are $3.7 \mathrm{~K}$ whereas that for $\mathrm{S} 2$ is $8.4 \mathrm{~K}$ opening a wide temperature zone dominated by mixed phase dynamics.

We use Maxwell's relation $\Delta S_{\text {Maxwell }}=\int_{0}^{H}(\partial M / \partial T)_{H} d H$ to estimate the entropy change from magnetization data both on increasing and decreasing fields. Typical behaviour is shown for sample S1 in figure 1(f). We obtain different values of entropy, much higher in increasing field, presenting giant peaks. However the most important fact to be observed is the striking similarity between the phase fraction derivatives (inset of figure 1(c)) and the calculated entropy on increasing field for all samples shown in figure 1(d) for 5 Tesla. The sharper phase derivative and higher entropy peaks are obtained for samples S1 and S3 while for S2, a smaller and slower but wider phase dynamics is observed.

To estimate a real entropy value without the mixed-phase influence, we model the magnetization $(M)$ of the system as $M=x M_{1}+(1-x) M_{2}$ for $H\left\langle H_{C}(T)\right.$ and $M=M_{1}$ for $\left.H\right\rangle H_{C}(T)$, where $x$ is taken as a function of temperature only, $M_{1}, M_{2}$ are the magnetization of ferromagnetic and paramagnetic phases, respectively and $H_{C}$ is the critical field of the phase transition. The entropy change up to $H\left\langle H_{C}(T)\right.$ can be formulated as [18]

$$
\Delta S_{c a l}=\frac{d}{d T} \int_{0}^{H}\left[x M_{1}+(1-x) M_{2}\right] d H=\frac{\partial x}{\partial T} \int_{0}^{H}\left(M_{1}-M_{2}\right) d H+\Delta S_{a v g}
$$


where $\Delta S_{\text {avg }}=x \int_{0}^{H} \frac{\partial M_{1}}{\partial T} d H+(1-x) \int_{0}^{H} \frac{\partial M_{2}}{\partial T} d H$.

Here, $\Delta S_{\text {avg }}$ is the weighted contribution of the ferro- and paramagnetic phase in the system, while the first term of equation(1) represents the main effect from nonequilibrium, and as such is a non-physical contribution to the entropy calculation, which should be removed. To obtain the entropy change to a field $H>H_{C}(T)$, the temperature dependence of $H_{C}(T)$ plays an important role (leading to the latent heat contribution). The total entropy change is then

$$
\begin{aligned}
\Delta S_{c a l} & =\frac{\partial}{\partial T} \int_{0}^{H_{C}(T)}\left[x M_{1}+(1-x) M_{2}\right] l H+\frac{\partial}{\partial T} \int_{H_{C}(T)}^{H} M_{1} d H \\
= & \frac{\partial x}{\partial T} \int_{0}^{H_{c}(T)}\left(M_{1}-M_{2}\right) d H+(1-x) \frac{\partial}{\partial T} H_{C}\left[M_{2}-M_{1}\right]+\Delta S_{a v g}+\int_{H_{C}}^{H} \frac{\partial M_{1}}{\partial T} d H
\end{aligned}
$$

The first term here is again the non-physical contribution, the $2^{\text {nd }}$ represents the fraction (1-x) of the entropy coming from latent heat contribution (measured in the calorimetric experiment in the region of mixed state, since a fraction $\mathrm{x}$ is already in the FM state at zero field) and the last two terms are from magnetic contribution only.

For both cases, the contribution due to the temperature dependence of $\mathrm{x}\left(1^{\text {st }}\right.$ term in equations 1 and 2) represents the non-equilibrium effects. This contribution to the entropy calculation can be removed by calculating the $\int_{0}^{H}\left(M_{1}-M_{2}\right) d H$ integral, with the upper limit of the integral being the lesser value of $\mathrm{H}$ and $\mathrm{Hc}(\mathrm{T})$, and multiplying it by $\frac{\partial x}{\partial T}$. The integral is found to be temperature independent in the mixed-phase range. The calculation of entropy can be graphically represented in an entropy change vs $\frac{\partial x}{\partial T}$ plot, as shown in figure 1 e). A linear extrapolation of each point to $\frac{\partial x}{\partial T}=0$ using a slope equivalent to the calculation of the previous integral, will remove the non-physical contribution, resulting in a realistic entropy change estimation. The value of this (constant) slope can also be obtained from the regions of clear linearity in the entropy change vs $\frac{\partial x}{\partial T}$ plot. This graphical representation of the method will be seen in a clearer way when applied to the simulated data, presented later. Although this corrected entropy does not lead to the thermodynamic equilibrium value, it removes the major nonphysical term of the entropy change calculation by considering the limiting condition of no mixed phase change in the system, and includes the effect of latent heat of the transformation. 
For all the samples studied here, the entropy change vs $\frac{\partial x}{\partial T}$ curves for different field changes, up to the critical field value, are approximately linear (inset of figure 1(e)) with an increasing slope of the curve with increasing field. Around the critical field and above, the effect due to latent heat comes into play, introducing extra terms in the entropy (equation 2) leading to a corresponding inflection in the behaviour (figure 1(e)). The corrected value of averaged entropy change is shown in figure 1(f), and includes the latent heat of the transformation. This entropy change is much less than that obtained from Maxwell's relation, demonstrating how the high values of magnetic entropy change near the transition temperature are overestimated due to phase mixing introducing an extra contribution. The actual entropy maximum of the present systems resulting from magnetic contribution only is $\sim 21$, 14.5, $22.4 \mathrm{~J} / \mathrm{kg} . \mathrm{K}$ for S1, S2 and S3 respectively at 5 Tesla, compared to values above $40 \mathrm{~J} / \mathrm{kg} . \mathrm{K}$ for all samples. Also, the entropy corrected in this way is much closer to the value obtained from the decreasing field curves (figure 1(b)), which show reduced mixed-phase behavior. However, this corrected entropy is always less than the value in equilibrium condition. This is because we deal with a fraction (1-x) of the phase $\mathrm{M}_{2}$ remaining to transform which will give a fraction of entropy due to latent heat (equation 2) since part ( $\mathrm{x}$ ) of phase is already transformed at zero field. This average entropy change weighted by the fraction of each phase present, can be measured in calorimetric experiments. We regard $\mathrm{x}$ and its temperature dependence $\mathrm{dx} / \mathrm{dT}$ as parameters that can be externally manipulated by changing the measurement condition or the sample history and should therefore be carefully considered in the data analysis procedure, in order to obtain the true entropy calculation.

To understand in a controlled way the results obtained, a theoretical simulation of mixed-state first-order phase transition system was performed using the Bean-Rodbell model [19]. The details of the simulation parameters and methodology are described in [18]. The phase fraction $x(T)$ of the system is chosen to be a relatively broad and smooth sigmoid function with a dx/dT curve of $3 \mathrm{~K}$ FWHM, shown in figure 2(a). The isotherms near $T_{C}$ obtained are shown in the inset of figure 2(a). The magnetic entropy change estimated with Maxwell's relation results in a magnetocaloric peak effect exceeding the magnetic maximum of $\mathrm{Nk}_{\mathrm{B}}(2 \mathrm{~J}+1)$ (figure 2(b)), but, more importantly, presents a similar shape to the $\mathrm{dx} / \mathrm{dT}$ vs $\mathrm{T}$ curve, confirming a direct relation between the peak effect and the mixed-phase conditions (figure 2(a) and figure 2(b)). The plot of $\Delta \mathrm{S}$ as a function of $\mathrm{dx} / \mathrm{dT}$ is shown in the inset of figure 2(b) and a linear extrapolation to null values of dx/dT leads to the actual entropy 
value (figure 2(b)) which is again much lower than that obtained from Maxwell's relation on nonequilibrium magnetization. This theoretical simulation predicts the behaviour of entropy with phase fraction in a similar way as that obtained from experimental data, with clear regions of a constant entropy change versus dx/dT slope. These calculations support our previous analysis of experimental data.

In conclusion, our results, for the $1^{\text {st }}$ time, allow a realistic estimation of magnetic entropy change solely from experimental mixed phase magnetization data. By removing the mixed-phase state dynamic contribution to the results from Maxwell's relation, we obtain entropy change values that match well with experimental calorimetric data [14]. This work therefore gives a practical way to use already available nonequilibrium magnetization data and obtain a correct value of actual entropy change of first-order system with metamagnetic transitions, with no need to perform time consuming heat capacity measurements or measuring isofield $\mathrm{M}$ versus $\mathrm{T}$ magnetization studies.

The authors thank D. Rocco for sample preparation and S. Gama and R. Burriel for valuable discussions.

The authors acknowledge the financial support from FCT through Project No. CERN/FP/83643/2008, grants No. SFRH/BPD/39262/2007 (S. Das), SFRH/BD/17961/2004 and SFRH/BPD/63942/2009 (J.S. Amaral). 


\section{References}

[1] V. K. Pecharsky, K. A. Gschneidner Jr., Phys. Rev. Lett. 78, 4494 (1997)

[2] L. Tocado, E Palacios, R Burriel, J Therm. Anal. Calorim. 84, 213 (2006)

[3] O Tegus, E Bruck, K H J Buschow, F R de Boer, Nature (London) 415, 150 (2002)

[4] A Fujita, S Fujieda, Y Hasegawa, K Fukamichi, Phys. Rev. B 67, 104416 (2003); H. Wada, T. Morikawa, K. Taniguchi, T. Shibata, Y. Yamada, and Y. Akishige, Physica B 328, 114 (2003)

[5] A. de Campos, D. L. Rocco, A. M. G. Carvalho, L. Caron, A. A. Coelho, S. Gama, L. M. Da Silva, F. C. G. Gandra, A. O. dos Santos, L. P. Cardoso, P. J. von Ranke and N. A. de Oliveira, Nat. Mater. 5, 802 (2006)

[6] D. L. Rocco, A. de Campos, A. M. G. Carvalho, L. Caron, A. A. Coelho, S. Gama, F. C. G. Gandra, A. O. dos Santos, L. P. Cardoso, P. J. von Ranke and N. A. de Oliveira, Appl. Phys. Lett. 90, 242507 (2007)

[7] S. Gama, A. A. Coelho, A. de Campos, A. M. G. Carvalho, F. C.G. Gandra, P. J. von Ranke and N. A. de Oliveira, Phys. Rev. Lett. 93, 237202 (2004)

[8] J-D Zou, B-G Shen, B. Gao, J. Shen, and J-R. Sun, Advanced Materials 21, 693 (2009)

[9] D. Liu, M. Yue, J. Zhang, T. M. McQueen, J. W. Lynn, X. Wang, Y. Chen, J. Li, R. J. Cava, X. Liu, Z. Altounian, and Q. Huang, Phy. Rev. B 79, 014435 (2009)

[10] N. K. Sun, W. B. Cui, D. Li, D. Y. Geng, F. Yang, and Z. D. Zhang, Appl Phys Lett 92, 072504 (2008)

[11] C. L. Zhang, D. H. Wang, Q. Q. Cao, Z. D. Han, H. C. Xuan, and Y. W. Du, Appl Phys Lett 93, 122505 (2008)

[12] A. L. Lima Sharma, P. A. Sharma, S. K. McCall, S.-B. Kim and S.-W. Cheong, Appl Phys Lett 95, 092506 (2009)

[13] G J Liu, J R Sun, J Shen, B Guo, H W Zhang, F X Hu, B G Shen, Appl Phys Lett 90, 032507 (2007)

[14] L Tocado, E Palacios, R Burriel, J Appl Phys 105, 093918 (2009)

[15] V K Pecharsky, K A Gschneidner Jr, J Appl Phys 86, 6315 (1999)

[16] A. Giguère, M. Foldeaki, B. Ravi Gopal, R. Chahine, T. K. Bose, A. Frydman, and J. A. Barclay, Phys. Rev. Lett. 83, 2262 (1999)

[17] J S Amaral, V S Amaral, Appl Phys Lett 94, 042506 (2009)

[18] J. S. Amaral, V.S. Amaral, J. Magn. Magn. Mater. (2009), doi:10.1016/j.jmmm.2009.06.013

[19] C P. Bean and D. S. Rodbell, Phys. Rev. 126, 104 (1962) 

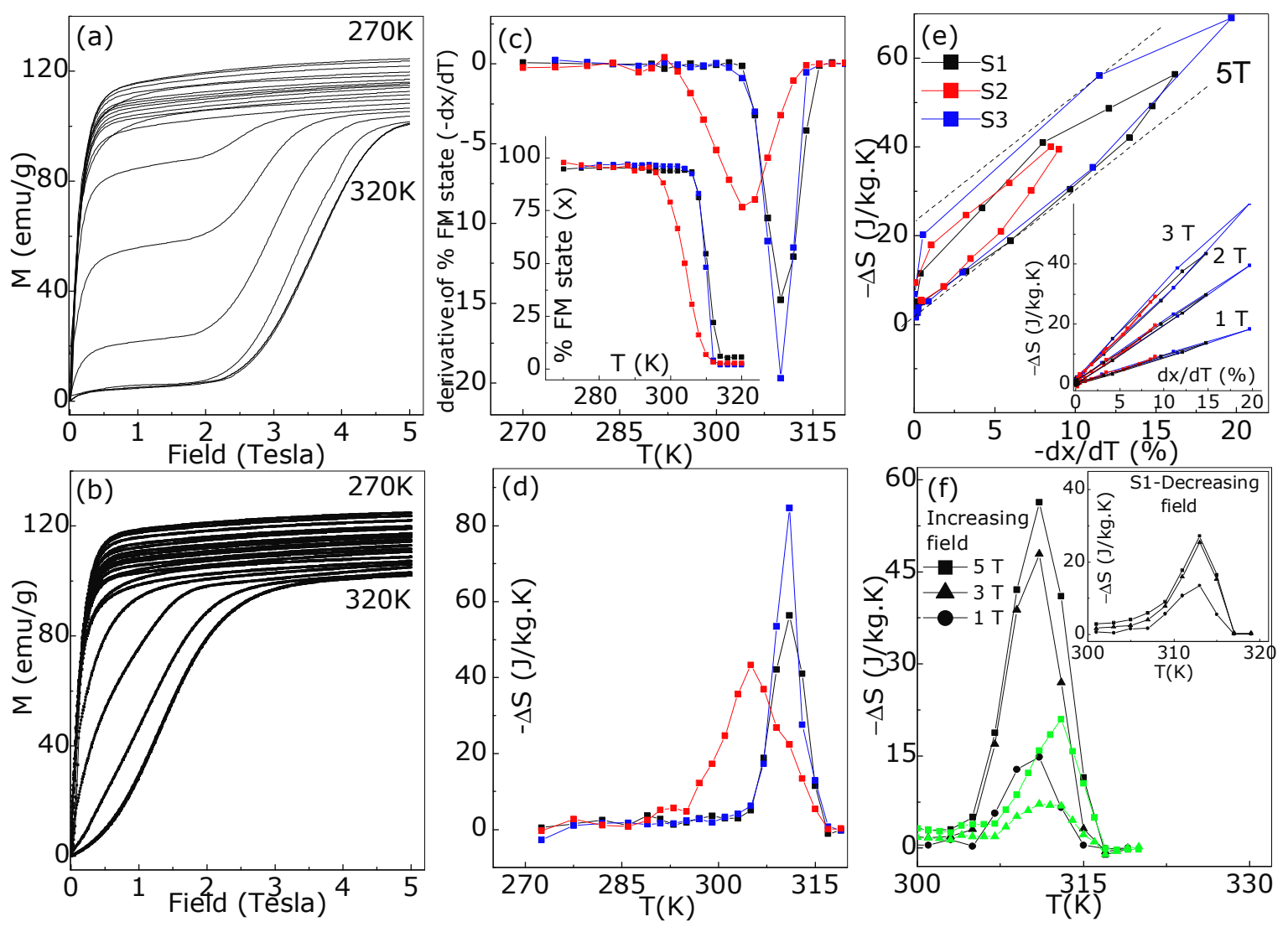

Figure 1. Isothermal magnetization curve of sample $\mathrm{S} 1$ from $270 \mathrm{~K}$ to $320 \mathrm{~K}$ shown up to 5 Tesla for (a) increasing and (b) decreasing field conditions, measured in the range 0-10 Tesla with $2 \mathrm{~K}$ temperature interval; (c) Temperature dependence of the derivative of the ferromagnetic phase fraction of the samples, inset shows the FM fractions; (d) magnetic entropy change $\Delta \mathrm{S}$ of the samples at 5 Tesla field obtained with Maxwell's relation for increasing field; (e) variation of $\Delta \mathrm{S}$ with FM fraction for the samples at 5 Tesla field, inset at 1, 2 and 3 Tesla, the slope of the dashed lines, used for extrapolation, results from calculations described in the text; (f) $\Delta \mathrm{S}$ of the sample S1 for increasing field, green lines result from corrections at 3 and 5 Tesla, inset shows $\Delta \mathrm{S}$ for decreasing field. The colour symbols in (e) apply for all the figures. 

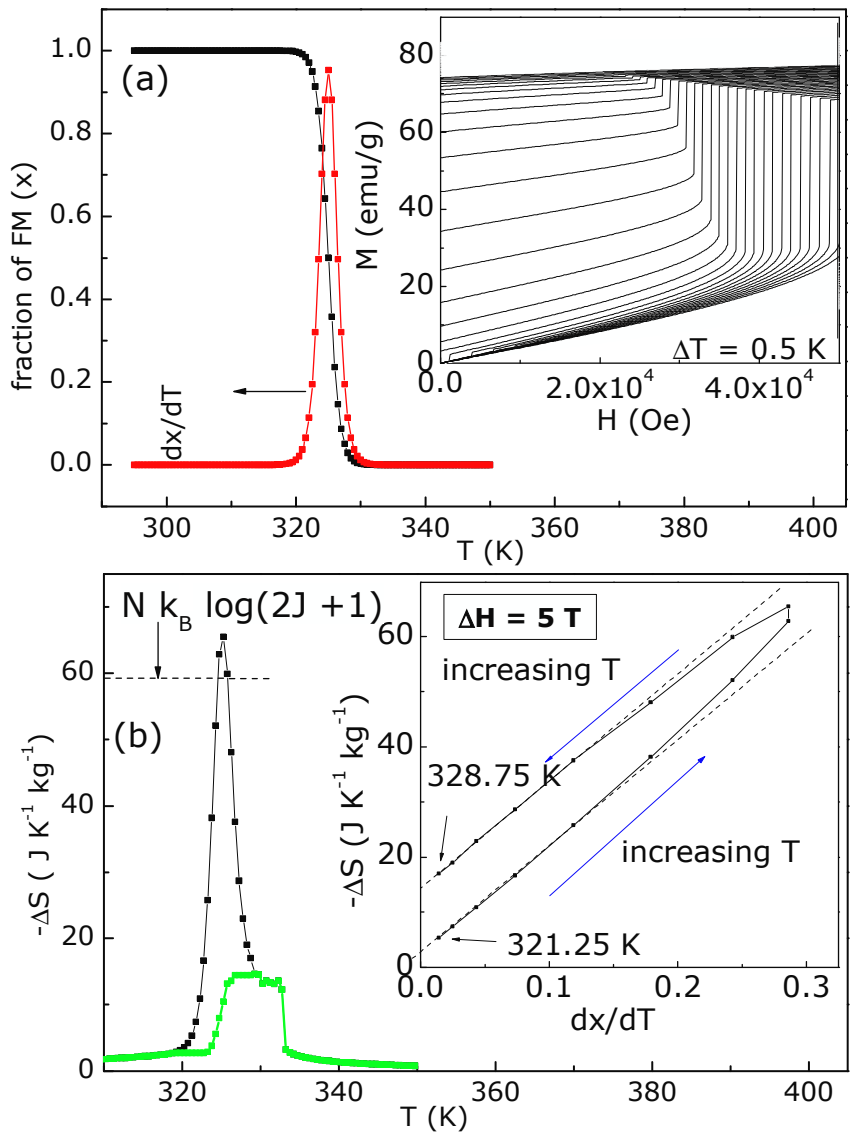

Figure 2 (a) Sigmoid function chosen for the FM phase fraction of the $1^{\text {st }}$ order system for simulation and its temperature derivative; inset shows the $\mathrm{M}$ vs $\mathrm{H}$ isotherms from Bean-Rodbell model incorporating the phase fraction, (b) entropy vs. T from Maxwell's relation, green line results from correcting phase transformation effect; inset is the entropy change vs. phase fraction from the simulation, where the slope of the dashed lines, used for extrapolation, results from calculations described in the text. 